\title{
Erratum: Stringy stability of charged dilaton black holes with flat event horizon
}

\author{
Yen Chin Ong ${ }^{b, c}$ and Pisin Chen ${ }^{a, b, c, d}$ \\ ${ }^{a}$ Graduate Institute of Astrophysics, National Taiwan University, \\ Taipei, 10617 Taiwan \\ ${ }^{b}$ Department of Physics, National Taiwan University, \\ Taipei, 10617 Taiwan \\ ${ }^{c}$ Leung Center for Cosmology and Particle Astrophysics, National Taiwan University, \\ Taipei, 10617 Taiwan \\ ${ }^{d}$ Kavli Institute for Particle Astrophysics and Cosmology, SLAC National Accelerator Laboratory, \\ Stanford University, \\ Stanford, CA 94305, U.S.A. \\ E-mail: d99244003@ntu.edu.tw, pisinchen@phys.ntu.edu.tw
}

ERRATUM TO: JHEP08(2012)079

ARXIV EPRINT: 1205.4398 
There were some confusions regarding whether some discussions are about electrically charged dilaton black holes or a magnetically charged one. We wish to clarify that eq. (2.3) is the metric of a magnetically charged black hole. If the black hole is electrically charged, then the string metric is different:

$$
g(\text { string })=-\left(1-\frac{2 M}{r}\right)\left(1-\frac{Q^{2}}{M r}\right) d t^{2}+\frac{\left(1-\frac{Q^{2}}{M r}\right)}{\left(1-\frac{2 M}{r}\right)} d r^{2}+r^{2}\left(1-\frac{Q^{2}}{M r}\right)^{2} d \Omega^{2} .
$$

Under the electromagnetic duality, $\phi \rightarrow-\phi$ as we map electrically charge black hole solution to a magnetically charged one, thus the string coupling for the electrical charged hole remains small near an extremal horizon. This is unlike the magnetically charged case, in which the string coupling diverges in the same limit.

In this paper we are only concerned with the electrically charged holes, therefore our conclusion remains unchanged.

However it must be noted that in the discussion section [specifically, page 17 and 18], the sentences:

"This is of course, subjected to the caveat that due to eq. (2.4), string coupling near extremal horizon becomes very large, thus signaling the failure of semiclassical treatment of gravity. Higher order corrections to gravity becomes necessary if we want to be confident about the properties of extremal charged dilaton black holes."

and

"Higher order corrections to gravity becomes necessary if we want to be confident about the properties of extremal charged dilaton black holes."

refer, of course, to the magnetically charged black holes with spherical topology. In addition, strictly speaking they are statements regarding asymptotically flat case, and should be checked explicitly for asymptotically AdS black holes.

Open Access. This article is distributed under the terms of the Creative Commons Attribution License (CC-BY 4.0), which permits any use, distribution and reproduction in any medium, provided the original author(s) and source are credited. 